\title{
MULTI-PARAMETRIC ANALYSIS OF THE LOWEST NATURAL FREQUENCIES OF STRONGLY INHOMOGENEOUS ELASTIC RODS
}

\author{
Julius Kaplunov*, Danila A. Prikazchikov, Olga Sergushova \\ Keele University, Keele, Staffordshire, ST5 5BG, UK
}

\begin{abstract}
The results of a multi-parametric analysis of the near-rigid body motions of a three-component strongly inhomogeneous elastic rod are presented. It is demonstrated that the values of the associated lowest natural frequencies tend to zero at large/small ratios of material and geometric parameters. The low-frequency behavior is classified into global and local regimes and the general conditions supporting global low-frequency regimes are derived. As an example, a rod with piecewise uniform properties is considered. A perturbation procedure oriented to a more general setup is developed.

Keywords: composite rod, contrast, low frequency
\end{abstract}

\section{Introduction}

Vibrations of inhomogeneous elastic structures find numerous applications in modern engineering, see e.g. [1, 2] among other contributions. In many cases structural components parts may have contrast material and geometric characteristics, including stiffness, volume density and size. As a typical example, we mention sandwich plates which are widely used in civil and mechanical engineering, and are therefore intensively studied, see e.g. [3, 4, [5], and references therein. The number of potential applications may increase significantly due to

\footnotetext{
*Corresponding author

Email address: j.kaplunov@keele.ac.uk (Julius Kaplunov )
} 
a rapidly growing area of metamaterials, see e.g. [6, 7].

This paper is concerned with investigation of low frequency vibrations of elastic rods composed of parts with contrast properties. It is observed that under certain restrictions on the material parameters and lengths the lowest natural frequencies become small, tending to zero at the limit of large/small contrasts.

The analysis is carried out for free vibrations of a non-uniform three-component rod. The basic problem parameters involve the ratios of the Young's moduli, densities and lengths of the components. A multi-parametric treatment reveals two types of low-frequency motions. One of these is the so-called global low-frequency regime corresponding to quasi-static behavior of all of the rod's components. Another type may be referred to as local low-frequency regime describing quasi-static behavior of "stronger", in particular, stiffer components and not preventing oscillating profiles in "weaker" parts, which is similar to recent results for homogenization of contrast periodic media, see [8, 9].

In the paper the attention is first drawn to the model problem of low25 frequency vibrations of a symmetric piecewise uniform rod with free ends, allowing a straightforward analytical treatment. The conditions on the parameters supporting the global low-frequency regime are derived. The associated displacement profile is approximated at leading order by linear functions. This agrees with an intuitive expectation, that the "stronger" parts perform almost 30 rigid body motion.

The consideration is then extended to the local low-frequency behaviour, along with other types of boundary conditions supporting global low-frequency regime. The cases which do not allow global low frequency modes are mentioned. Finally, the analysis is generalized to a non-uniform three-component 35 rod with variable material parameters depending on the longitudinal coordinate. Since a straightforward analytical approach is no longer possible, a perturbation scheme is developed leading to estimates for low natural frequencies and the displacement profile.

The developed methodology is not restricted to the three-component rod 
40 considered in the paper. It has a potential to be extended for a more sophisticated setup. In particular the obtained results could be applied to analysis of the lowest cut-off frequencies [10, 11] of sandwich plates and shells and also have some more general implications to 2D and 3D dynamic problems for elastic structures with contrast material parameters. In the latter case the perturbation

45 scheme presented in this paper may need to be amended by including numerical routines.

\section{Problem statement}

\begin{tabular}{lllll}
\hline \hline$-h_{3}-h_{1}$ & $-h_{1}$ & 0 & $h_{1}$ & $h_{2}+h_{1}$
\end{tabular}

Figure 1: Composite rod.

Consider time-harmonic vibrations of an elastic rod composed of three inhomogeneous parts. The axis $O x$ is chosen such that the origin $O$ is located ${ }_{50}$ in the middle of the inner part. The rod is finite, with the outer parts having free or fixed ends, and continuity assumed between the components. The inner part of the rod occupies the region $|x| \leq h_{1}$, with the outer parts specified by $-h_{1}-h_{3} \leq x \leq-h_{1}$ and $h_{1} \leq x \leq h_{1}+h_{2}$.

The equations of motion are written in the form

$$
\frac{d}{d x}\left(E_{i} \frac{d u}{d x}\right)+\rho_{i} \omega^{2} u=0, \quad i=1,2,3,
$$

where $u$ is the displacement, $E_{i}$ are the Young's moduli, $\rho_{i}$ are the material

55 densities, $c_{i}=\sqrt{E_{i} / \rho_{i}}$ are the longitudinal wave speeds and $\omega$ is the vibration frequency. Here the indices 1, 2 and 3 correspond to the inner, right outer and left outer parts, respectively. 
The continuity conditions at the interfaces are written as

$$
\begin{aligned}
u\left(-h_{1}+0\right) & =u\left(-h_{1}-0\right), \\
u\left(h_{1}-0\right) & =u\left(h_{1}+0\right), \\
E_{1} u^{\prime}\left(-h_{1}+0\right) & =E_{3} u^{\prime}\left(-h_{1}-0\right), \\
E_{1} u^{\prime}\left(h_{1}-0\right) & =E_{2} u^{\prime}\left(h_{1}+0\right) .
\end{aligned}
$$

We consider three types of boundary conditions on the outer ends, namely free ends

$$
\begin{aligned}
u^{\prime}\left(-h_{1}-h_{3}\right) & =0, \\
u^{\prime}\left(h_{1}+h_{2}\right) & =0,
\end{aligned}
$$

fixed ends

$$
\begin{aligned}
u\left(-h_{1}-h_{3}\right) & =0, \\
u\left(h_{1}+h_{2}\right) & =0,
\end{aligned}
$$

and mixed boundary conditions

$$
\begin{aligned}
u\left(-h_{1}-h_{3}\right) & =0, \\
u^{\prime}\left(h_{1}+h_{2}\right) & =0 .
\end{aligned}
$$

Let us represent the variable material parameters as

$$
E_{i}=E_{i}^{*} \tilde{E}_{i}(x), \quad \rho_{i}=\rho_{i}^{*} \tilde{\rho}_{i}(x), \quad c_{i}=c_{i}^{*} \tilde{c_{i}}(x), \quad i, j=1,2,3
$$

where $E_{i}^{*}, \rho_{i}^{*}$ and $c_{i}^{*}$ are average values of the associated quantities. We also introduce dimensionless longitudinal variable

$$
\chi=\frac{x}{h_{1}},
$$

and scaled frequencies

$$
\lambda_{i}=\frac{\omega h_{i}}{c_{i}^{*}}, \quad i=1,2,3,
$$

along with the dimensionless parameters

$$
E_{i j}=\frac{E_{i}^{*}}{E_{j}^{*}}, \quad \rho_{i j}=\frac{\rho_{i}^{*}}{\rho_{j}^{*}}, \quad c_{i j}=\frac{c_{i}^{*}}{c_{j}^{*}}, \quad h_{i j}=\frac{h_{i}}{h_{j}}, \quad i, j=1,2,3 .
$$




\section{Antisymmetric vibrations of a rod with free ends}

Let us begin with a simple problem for a symmetric composite rod with constant material parameters. In this case due to symmetry of the rod

$$
E_{2}=E_{3}, \quad \rho_{2}=\rho_{3}, \quad c_{2}=c_{3}, \quad h_{2}=h_{3}, \quad \lambda_{2}=\lambda_{3},
$$

hence, the set of dimensionless parameters is reduced to

$$
E=\frac{E_{1}}{E_{2}}, \quad \rho=\frac{\rho_{1}}{\rho_{2}}, \quad c=\frac{c_{1}}{c_{2}}, \quad h=\frac{h_{1}}{h_{2}} .
$$

Consider now vibrations of a rod subject to free ends boundary conditions (3). Since the rod is symmetric, the analysis for $\chi \geq 0$ is sufficient for derivation of the frequency equation. The problem may be decomposed into symmetric and antisymmetric cases. Let us focus on the antisymmetric problem in this section, adopting for the displacement of the inner part

$$
u=A \sin \lambda_{1} \chi, \quad|\chi| \leq 1,
$$

following from Eq.(11). The displacement of the outer part is written in general form

$$
u=C \cos h \lambda_{2} \chi+D \sin h \lambda_{2} \chi, \quad 1 \leq|\chi| \leq 1+h^{-1} .
$$

Substituting Eq.(6) and Eq.(7) into Eq.(2) and Eq.(3), we arrive at

$$
\begin{gathered}
A \sin \lambda_{1}-C \cos h \lambda_{2}-D \sin h \lambda_{2}=0, \\
A \frac{E}{c} \cos \lambda_{1}+C \sin h \lambda_{2}-D \cos h \lambda_{2}=0, \\
-C \sin \left(h \lambda_{2}+\lambda_{2}\right)+D \cos \left(h \lambda_{2}+\lambda_{2}\right)=0 .
\end{gathered}
$$

A linear algebraic system Eq.(8) possesses non-trivial solutions provided that the associate determinant vanishes, leading to the frequency equation

$$
\tan \lambda_{1} \tan \lambda_{2}=\frac{E}{c} \text {. }
$$

Using Eq.(8), we can represent the displacement profile as

$$
\begin{array}{lll}
u=\sin \lambda_{1} \chi, & & |\chi| \leq 1, \\
u=\sin \lambda_{1} \cos h \lambda_{2}(\chi-1)+\frac{E}{c} \cos \lambda_{1} \sin h \lambda_{2}(\chi-1), & & 1 \leq|\chi| \leq 1+h^{-1} .
\end{array}
$$


The studied low-frequency motion may be split into cases of global low-

60 frequency regime $\left(\lambda_{1} \ll 1, \lambda_{2} \ll 1\right)$, and local low-frequency behaviour $\left(\lambda_{1} \ll 1\right.$, $\lambda_{2} \gtrsim 1$ or $\left.\lambda_{1} \gtrsim 1, \lambda_{2} \ll 1\right)$.

\subsection{Global low-frequency regime}

In case of $\lambda_{1} \ll 1$ and $\lambda_{2} \ll 1$, associated with quasi-static behaviour of both inner and outer components of the rod, the frequency equation Eq.(9) reduces to the following approximate form

$$
\lambda_{1} \lambda_{2}=\frac{E}{c}
$$

from which we deduce

$$
\lambda_{1}=\sqrt{\rho h}
$$

and

$$
\lambda_{2}=\sqrt{\frac{E}{h}} .
$$

Formulas (12) and (13) combined with strong inequalities $\lambda_{1} \ll 1, \lambda_{2} \ll 1$ imply

$$
E \ll h \ll \rho^{-1},
$$

which gives an estimate for material parameters allowing global low-frequency regime.

Fig. 2 contains frequency curves depending on the relative thickness $h$, including the decreasing curve of the first mode for the outer part (shown by a dashed blue line) defined by Eq.(9), and the increasing curves for the first three modes for the inner part along with the asymptotic approximation Eq.12) depicted by dotted line. It can be seen from Fig. 2 that varying $h$ between $E$ 70 and $\rho^{-1}$, it is possible to find a range of values of $h$, such that $\lambda_{1}$ and $\lambda_{2}$ are both small. Good agreement between the first harmonic for $\lambda_{1}$ defined from Eq.(9) and its approximation Eq.(12) is also observed.

It may also be noticed that the distance between the first and second modes is substantial. This may have a serious implication for the dispersion analysis 75 of bending of sandwich plates composed of layers having contrast properties. 


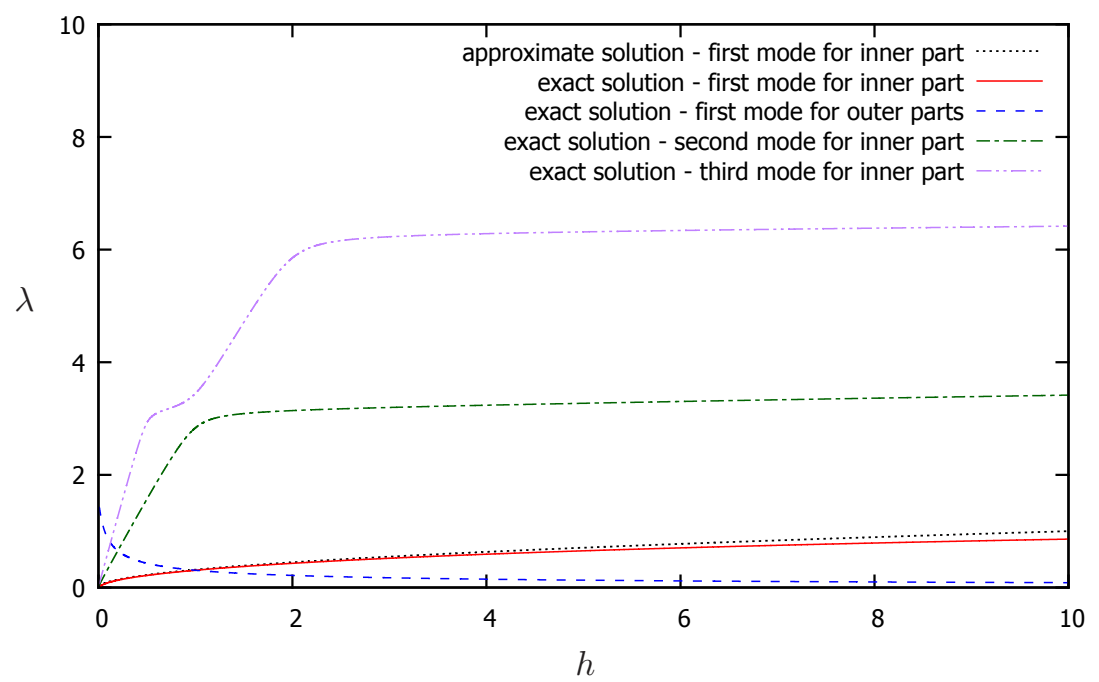

Figure 2: Frequency vs relative thickness $h: E=0.1, \rho=0.1$.

Indeed, the frequencies studied within 1D framework correspond to the cut-offfrequencies of the plate [10]. Therefore, one may expect that the first cut-offfrequency being small leads to the phenomena of weak coupling between the fundamental mode and the first harmonic, requiring special shear deformation 80 plate theories in the long-wave low-frequency region, see e.g. [12, 13, 14].

Introducing the dimensionless displacement $U=\frac{u}{\lambda_{1}}$, and noting that $\frac{E}{c} \ll 1$ from Eq. (11), and that the condition $0 \leq h \lambda_{2}(\chi-1) \leq \lambda_{2}$ holds true for the outer part, we arrive at the following approximate profile of the eigenform

$$
\begin{aligned}
& U=\chi, \quad|\chi| \leq 1 \\
& U=1, \quad 1 \leq|\chi| \leq 1+h^{-1}
\end{aligned}
$$

see Fig. 3.

It is readily observed from Fig. 3 that the "stronger" outer parts perform rigid body motion (at leading order), whereas the "weaker" inner part undergoes an almost homogeneous deformation.

It is also worth noting that in the vicinity of intersection of curves for $\lambda_{1}$ 


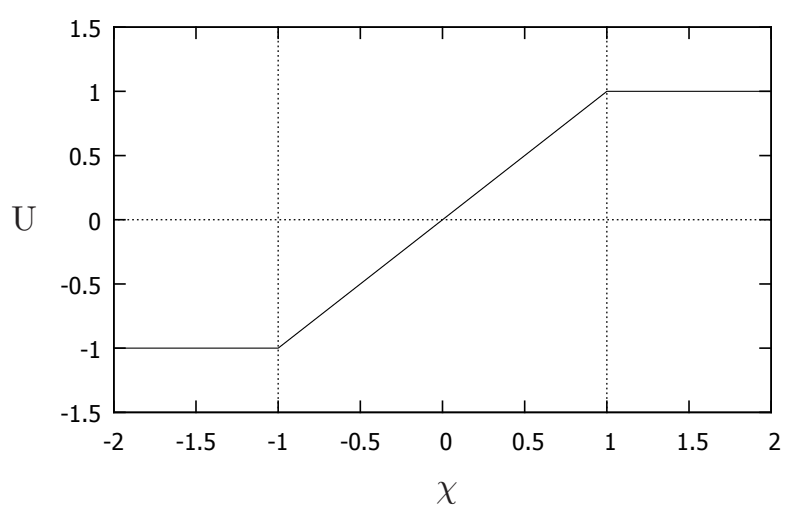

Figure 3: Displacement profile (15) (global low-frequency regime): $h=1$.

and $\lambda_{2}$ in Fig. 2, when $\lambda_{1}=\lambda_{2}=\lambda$, in the low frequency region

$$
\lambda=(E \rho)^{1 / 4}
$$

${ }_{85}$ The low-frequency analysis is therefore performed in respect of a small parameter $E \rho$, which may be interpreted physically as having a "weak" inner and "strong" outer components.

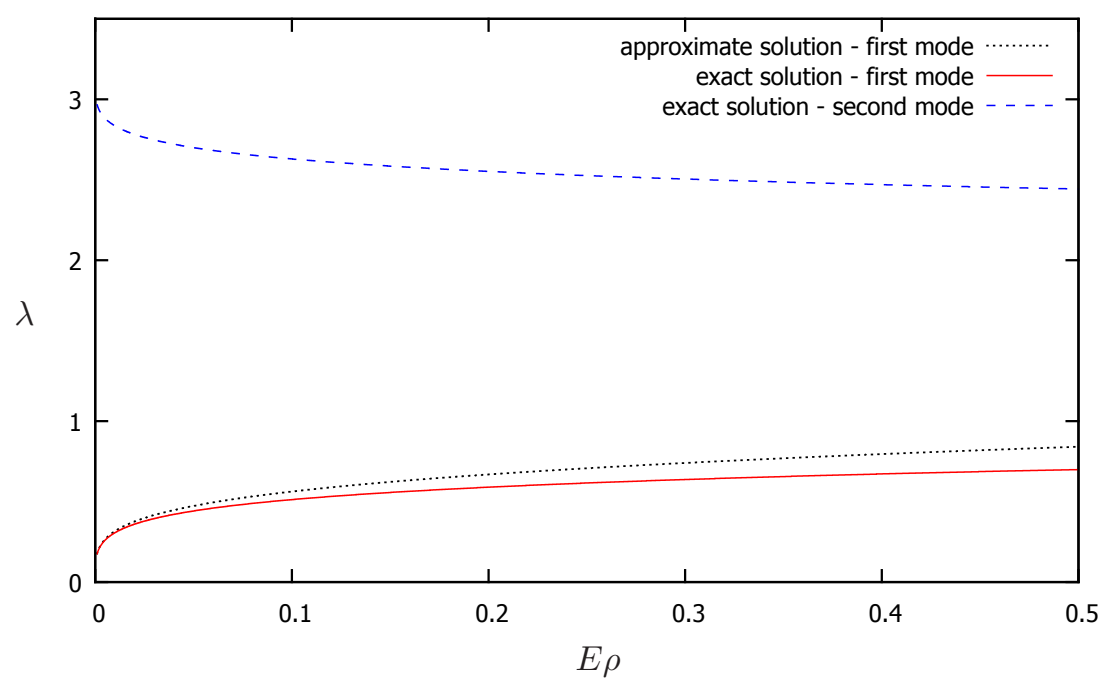

Figure 4: Variation of the frequency $\lambda$ vs the parameter $E \rho$ for antisymmetric vibrations of a rod with free ends.

It may be observed from Fig. 4 that the first mode of the frequency equation (9) decreases as $E \rho \rightarrow 0$, with the distance between the first and second 
90 modes increasing. Naturally, the accuracy of the asymptotic approximation (16) increases as $E \rho \rightarrow 0$.

\subsection{Local low-frequency regime for inner section}

Let us now consider the case of $\lambda_{1} \ll 1$ and $\lambda_{2} \gtrsim 1$, allowing wave-like phenomena in the outer parts and precluding those in the inner part of the rod. We note an underlying link with problems of homogenization of periodic contrast media, see e.g. [8, 15]. The frequency equation is then given by

$$
\tan \lambda_{2}=\frac{E}{h \lambda_{2}} \text {. }
$$

The corresponding scaled displacement takes the form

$$
\begin{array}{lll}
U=\chi, & & |\chi| \leq 1, \\
U=\cos h \lambda_{2}(\chi-1)+\frac{E}{h \lambda_{2}} \sin h \lambda_{2}(\chi-1), & 1 \leq|\chi| \leq 1+h^{-1} .
\end{array}
$$

Let us consider several particular cases.

Case 1. If $\frac{E}{h \lambda_{2}} \ll 1$, then from the frequency equation (17) $\tan \lambda_{2} \ll 1$, hence $\lambda_{2} \approx \pi n$, with $n=1,2,3, \ldots$. Noting that $\lambda_{1} \ll 1$ and $\frac{E}{h \lambda_{2}} \ll 1$, we deduce

$$
\frac{E}{h} \ll n \ll \frac{c}{h} .
$$

The displacement can be expressed as

$$
\begin{array}{ll}
U=\chi, & |\chi| \leq 1, \\
U=\cos \pi n h(\chi-1), & 1 \leq|\chi| \leq 1+h^{-1} .
\end{array}
$$

The resulting first two modes are shown on Fig. 5.

Case 2. If $\frac{E}{h \lambda_{2}} \gg 1$, then Eq.(17) implies $\lambda_{2} \approx \frac{\pi}{2}+\pi n$, with $n=0,1,2, \ldots$. Using the conditions $\lambda_{1} \ll 1$ and $\frac{E}{h \lambda_{2}} \gg 1$, we obtain

$$
n \ll \min \left[\frac{c}{h}, \frac{E}{h}\right] \text {. }
$$

The displacement profile is given by

$$
\begin{array}{ll}
U=\chi, & |\chi| \leq 1, \\
U=\cos \frac{\pi(2 n+1)}{2} h(\chi-1)+\frac{2 E}{h \pi(2 n+1)} \sin \frac{\pi(2 n+1)}{2} h(\chi-1), \quad 1 \leq|\chi| \leq 1+h^{-1} .
\end{array}
$$




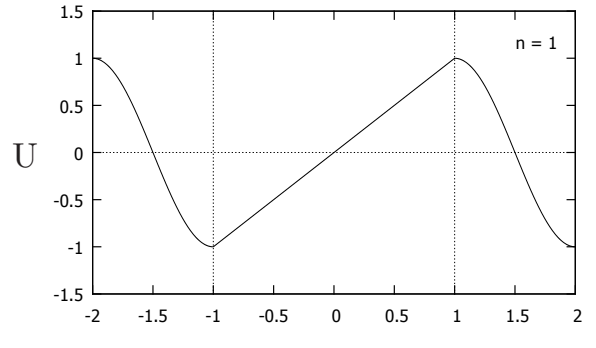

a)

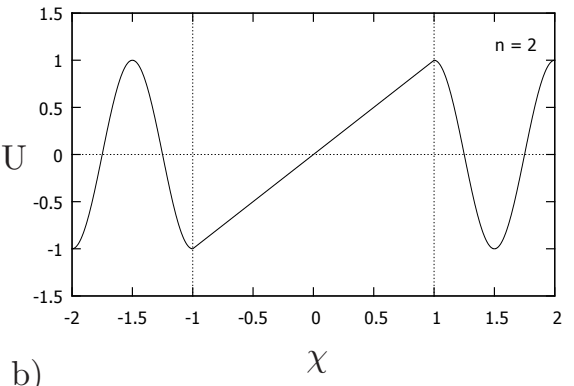

b)

Figure 5: Displacement profiles 20] (local low-frequency regime for inner part): $h=1$. a) $n=1$; b) $n=2$.

Note that since $\frac{E}{\lambda_{2} h} \gg 1$, for $\chi>1$

$$
U \sim \frac{2 E}{h \pi(2 n+1)} \sin \frac{\pi(2 n+1)}{2} h(\chi-1),
$$

95 with the first term in Eq.(22) acting similarly to a boundary layer, smoothing the discontinuity at $\chi=1$.

The overall profile for the first two modes of the displacement is presented on Fig. 6.

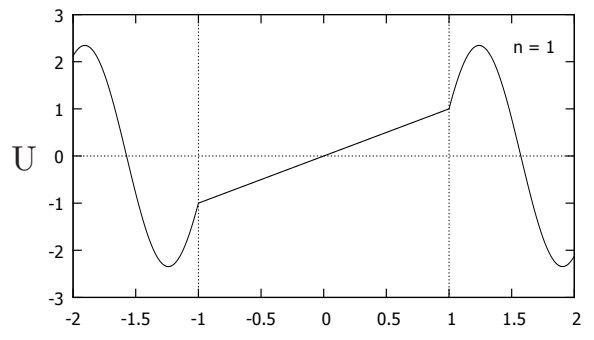

a)

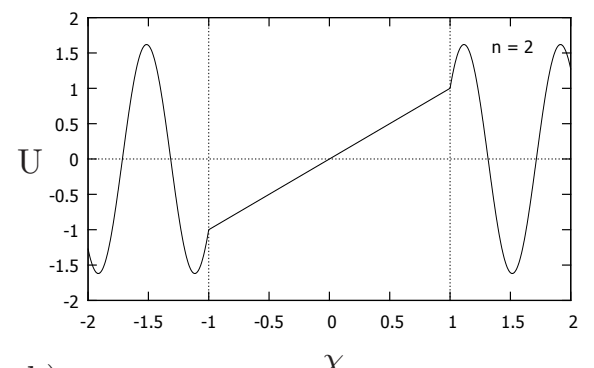

b)

Figure 6: Displacement profiles 22] (local low-frequency regime for inner part): $h=1$, $E=10$. a) $n=1$; b) $n=2$.

Case 3. When $\frac{E}{h \lambda_{2}} \sim 1$, then the conditions for $\lambda_{2}$ reduce to

$$
\lambda_{2} \sim \frac{E}{h} \ll \frac{c}{h} .
$$




\subsection{Local low-frequency regime for outer sections}

Let us consider another case of local low-frequency regime, when $\lambda_{1} \gtrsim 1$ and $\lambda_{2} \ll 1$. The frequency equation is then written as

$$
\tan \lambda_{1}=\frac{\rho h}{\lambda_{1}},
$$

and the corresponding natural form is given by

$$
\begin{array}{ll}
u=\sin \chi \lambda_{1}, & |\chi| \leq 1, \\
u=\sin \lambda_{1}+E \lambda_{1}(\chi-1) \cos \lambda_{1}, & 1 \leq|\chi| \leq 1+h^{-1} .
\end{array}
$$

Similarly to the previous subsection the analysis is split into several cases.

Case 1. If $\frac{\rho h}{\lambda_{1}} \ll 1$, then $\tan \lambda_{1} \ll 1$, hence $\lambda_{1} \approx \pi n$, with $n=1,2,3 \ldots$. Using $\lambda_{2} \ll 1$ and $\frac{\rho h}{\lambda_{1}} \ll 1$, we infer

$$
\rho h \ll n \ll \frac{h}{c} .
$$

The displacement is expressed as

$$
\begin{array}{ll}
u=\sin \pi n \chi, & |\chi| \leq 1, \\
u=(-1)^{n} \operatorname{E} \pi n(\chi-1), & 1 \leq|\chi| \leq 1+h^{-1},
\end{array}
$$

see Fig. 7 for numerical illustrations of the first two modes.

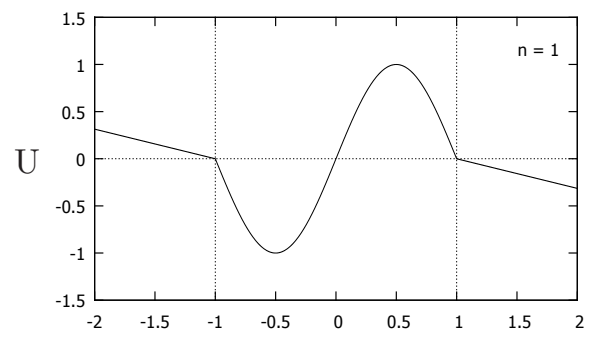

a)

Figure 7: Displacement profiles (28) (local low-frequency regime for outer parts): $h=1$, $E=0.1$. a) $n=1$; b) $n=2$.

Case 2. If $\frac{\rho h}{\lambda_{1}} \gg 1$, then from (25) $\lambda_{1} \approx \frac{\pi}{2}+\pi n$, with $n=0,1,2 \ldots$ The conditions $\lambda_{2} \ll 1$ and $\frac{\rho h}{\lambda_{1}} \gg 1$ give

$$
n \ll \min \left[\rho h, \frac{h}{c}\right] .
$$


Then the displacement profile is

$$
\begin{array}{ll}
u=\sin \frac{\pi(2 n+1)}{2} \chi, & |\chi| \leq 1, \\
u=(-1)^{n}, & 1 \leq|\chi| \leq 1+h^{-1},
\end{array}
$$

see Fig. 8 .

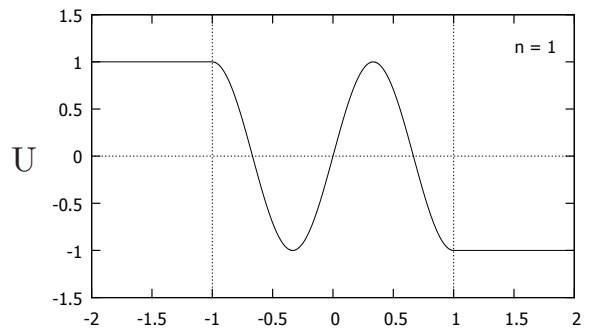

a)

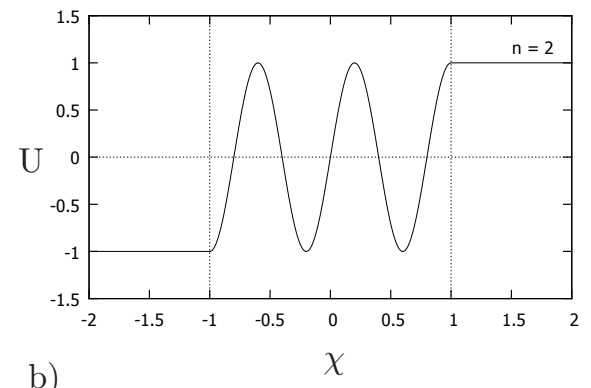

b)

Figure 8: Displacement profiles (30) (local low-frequency regime for outer parts): $h=1$. a) $n=1$; b) $n=2$.

In view of the conditions $\lambda_{1} \gtrsim 1, \lambda_{2} \ll 1$ it is expected to observe oscillatory behaviour for the inner section of the rod along with polynomial dependence for the outer components, which is confirmed by Figs. 7 and 8 .

Case 3. If $\frac{\rho h}{\lambda_{1}} \sim 1$, the conditions for $\lambda_{1}$, allowing low-frequency regime, can be obtained in the form

$$
\lambda_{1} \sim \rho h \ll \frac{h}{c} .
$$

\section{Further examples}

In this section we consider some other types of boundary conditions. We remark that cases of antisymmetric vibrations of a rod with fixed ends and symmetric vibrations of a rod with free ends do not allow global low-frequency regimes and are therefore excluded from consideration. 


\subsection{Symmetric vibrations of a rod with fixed ends}

Here we study the fixed ends boundary conditions (4), with the expression for the displacement of the inner part sought for as

$$
u=A \cos \lambda_{1} \chi, \quad|\chi| \leq 1,
$$

whereas the displacement of the outer part taken as Eq.(77).

Following a usual procedure, the frequency equation may be obtained in the form

$$
\cot \lambda_{1} \cot \lambda_{2}=\frac{E}{c} .
$$

The displacement profile is given by

$$
\begin{array}{lll}
u=\cos \lambda_{1} \chi, & & |\chi| \leq 1, \\
u=\cos \lambda_{1} \cos h \lambda_{2}(\chi-1)-\frac{E}{c} \sin \lambda_{1} \sin h \lambda_{2}(\chi-1), & & 1 \leq|\chi| \leq 1+h^{-1} .
\end{array}
$$

The low-frequency approximation for the frequency equation gives

$$
\lambda_{1} \lambda_{2}=\frac{c}{E},
$$

leading to

$$
\lambda_{1}=\sqrt{\frac{h}{E}}
$$

and

$$
\lambda_{2}=\frac{1}{\sqrt{\rho h}},
$$

with the conditions on material parameters corresponding to the global lowfrequency vibrations written as

$$
\rho^{-1} \ll h \ll E .
$$

The numerical illustrations of the dependence of $\lambda_{1}$ and $\lambda_{2}$ on $h$ in this case are very similar to that presented in Fig. 2, and are therefore omitted here.

The corresponding approximate eigenform is written as

$$
\begin{array}{ll}
u=1, & |\chi| \leq 1, \\
u=1-E \lambda_{1}^{2}(\chi-1), & 1 \leq|\chi| \leq 1+h^{-1},
\end{array}
$$




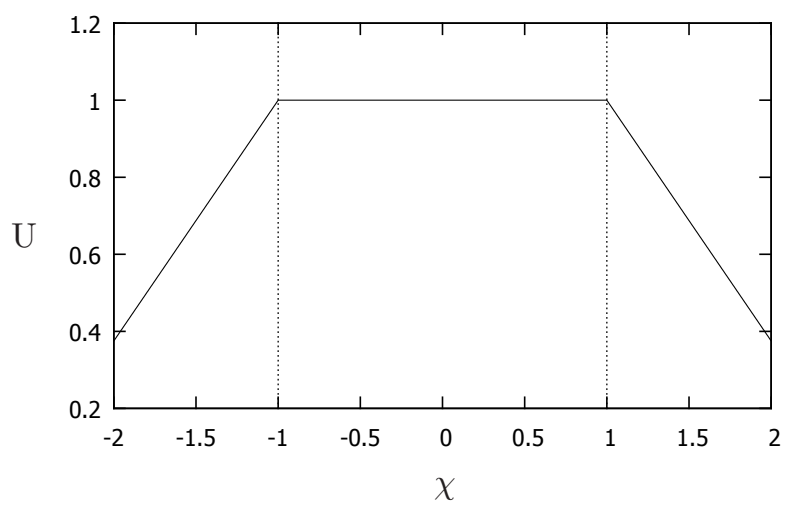

Figure 9: Displacement profile 39]: $h=1, E=10$.

see Fig. 9.

In the vicinity of $\lambda_{1}=\lambda_{2}=\lambda$, the approximate solution for $\lambda \ll 1$ is given by

$$
\lambda=(E \rho)^{-1 / 4}, \quad(E \rho \gg 1) .
$$

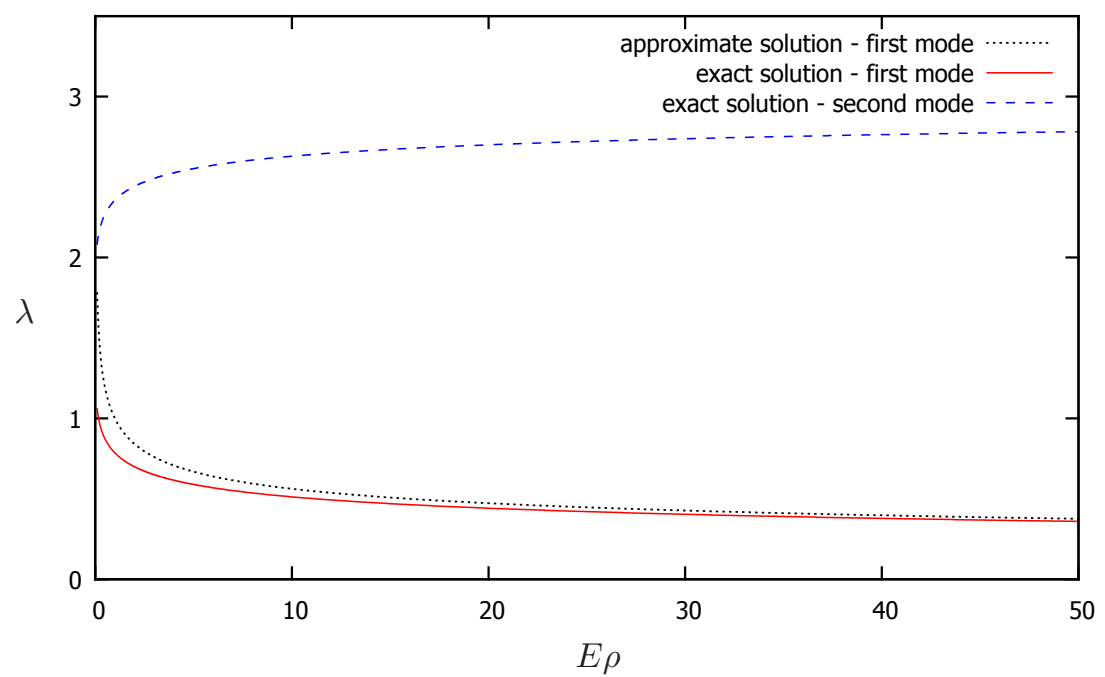

Figure 10: Dependence of the frequency $\lambda$ on the parameter $E \rho$ for symmetric vibrations of a rod with fixed ends.

As may be seen from Fig. 10, the first harmonic for $\lambda$ defined by Eq.(33) decreases as $E \rho \longrightarrow \infty$. The large values of $E \rho$ can be interpreted physically as a composite rod with a "strong" inner part and "weak" outer parts. 


\subsection{Vibrations of a rod with mixed boundary conditions}

Consider now vibrations of a rod subject to mixed boundary conditions Eq.(5). The problem now cannot be split into symmetric and anti-symmetric parts, so all three parts of the rod should be analysed. The displacement is sought for in the form

$$
\begin{array}{ll}
u=F \cos h \lambda_{2} \chi+G \sin h \lambda_{2} \chi, & -1-h^{-1} \leq \chi \leq-1, \\
u=A \cos \lambda_{1} \chi+B \sin \lambda_{1} \chi, & -1 \leq \chi \leq 1, \\
u=C \cos h \lambda_{2} \chi+D \sin h \lambda_{2} \chi, & 1 \leq \chi \leq 1+h^{-1} .
\end{array}
$$

Using Eq.(2) and Eq.(5) together with Eq.(41), the frequency equation is obtained in the form

$$
2 \cot 2 \lambda_{1} \cot 2 \lambda_{2}=\alpha+\frac{1}{\alpha}
$$

${ }_{120}$ where $\alpha=\frac{E}{c}$.

The displacement profile is then written as

$$
\begin{array}{ll}
u=\frac{\alpha \sin \lambda_{2}(h+1+h \chi)}{\beta}, & -1-h^{-1} \leq \chi \leq-1, \\
u=\frac{\sin \lambda_{1}(1+\chi) \cos \lambda_{2}+\alpha \cos \lambda_{1}(1+\chi) \sin \lambda_{2}}{\beta}, & -1 \leq \chi \leq 1, \\
u=\frac{\cos \lambda_{2}(h+1-h \chi)\left(\sin 2 \lambda_{1} \cos \lambda_{2}+\alpha \cos 2 \lambda_{1} \sin \lambda_{2}\right)}{\beta \cos \lambda_{2}}, & 1 \leq \chi \leq 1+h^{-1,}
\end{array}
$$

where

$$
\beta=\cos \lambda_{1} \cos \lambda_{2}-\alpha \sin \lambda_{1} \sin \lambda_{2}
$$

In the global low-frequency regime with $\lambda_{1} \ll 1$ and $\lambda_{2} \ll 1$, the approximation for the frequency equation is given by

$$
\frac{1}{2 \lambda_{1} \lambda_{2}}=\alpha+\frac{1}{\alpha}
$$

from which

$$
\lambda_{1}=\sqrt{\frac{h}{2\left(E+\rho^{-1}\right)}},
$$


and

$$
\lambda_{2}=\sqrt{\frac{h^{-1}}{2\left(E^{-1}+\rho\right)}} .
$$

Using the latter together with the inequalities $\lambda_{1} \ll 1$ and $\lambda_{2} \ll 1$ we deduce the following restrictions on the parameters

$$
\left(E^{-1}+\rho\right)^{-1} \ll h \ll E+\rho^{-1},
$$

allowing the global low-frequency regime.

The approximate profile of the displacement is given by

$$
\begin{array}{ll}
U=\frac{\gamma(h+1+h \chi)}{1-\gamma \lambda_{1}^{2}}, & -1-h^{-1} \leq \chi \leq-1, \\
U=\frac{1+\chi+\gamma}{1-\gamma \lambda_{1}^{2}}, & -1 \leq \chi \leq 1, \\
U=\frac{2+\gamma}{1-\gamma \lambda_{1}^{2}}, & 1 \leq \chi \leq 1+h^{-1},
\end{array}
$$

where $\gamma=\frac{E}{h}$, see Fig. 11 .

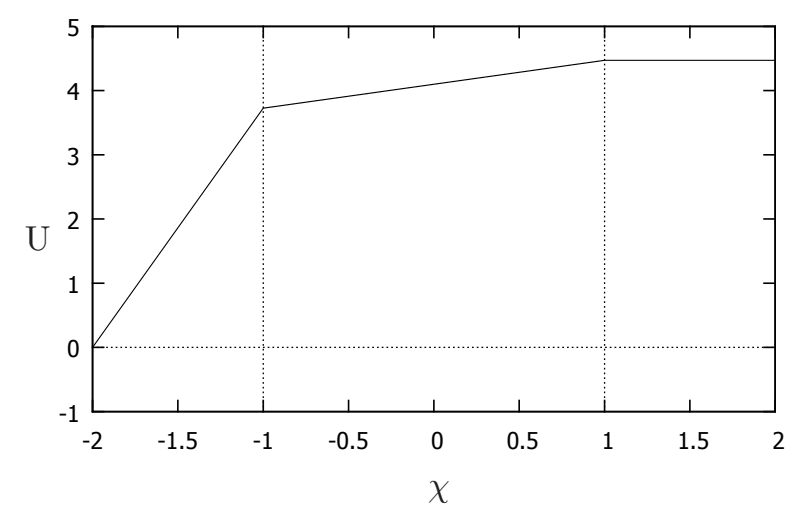

Figure 11: Displacement profile 48): $h=1, E=10$.

When $\lambda_{1}=\lambda_{2}=\lambda$, the approximate solution for $\lambda \ll 1$ is

$$
\lambda=\frac{(4 E \rho)^{1 / 4}}{\sqrt{E \rho+1}}, \quad\left(\sqrt{E \rho}+\sqrt{(E \rho)^{-1}} \gg 1\right) .
$$




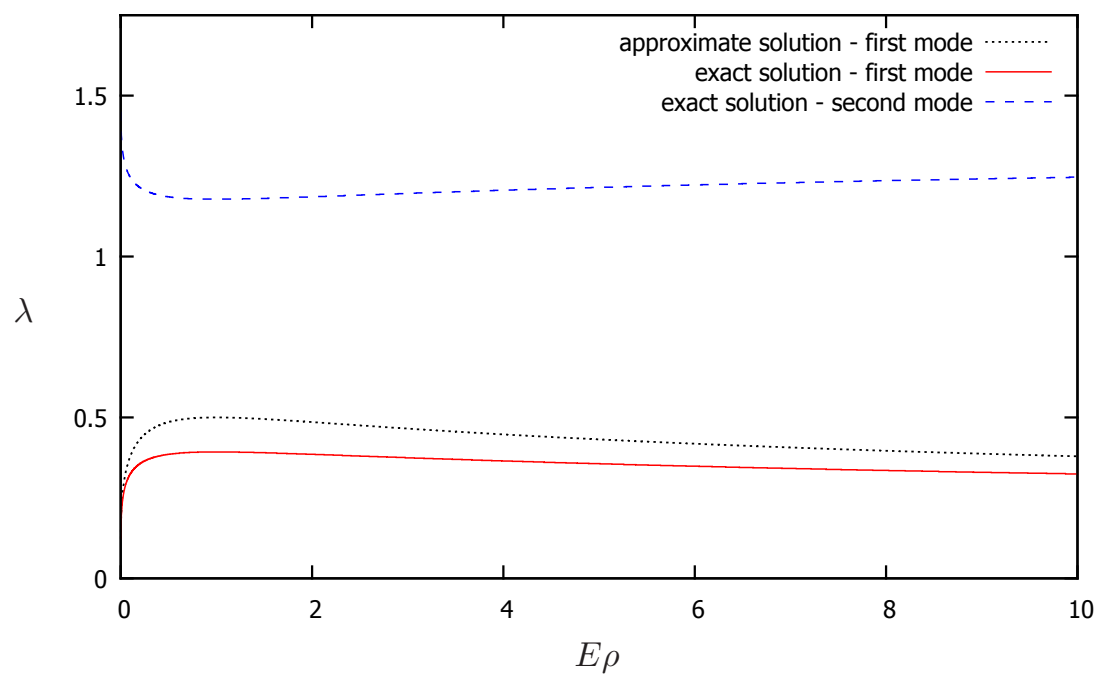

Figure 12: Variation of the frequency $\lambda$ vs the parameter $E \rho$ for the case of mixed boundary conditions.

Fig. 12 reveals the behaviour of the first and second modes of the frequency $\lambda$ governed by (42) as well as approximate solution for the first mode of (49). It is seen that the first mode decreases in both limits $E \rho \rightarrow 0$ and $E \rho \rightarrow \infty$, indicating possibilities of global low-frequency vibrations in a rod with mixed boundary conditions for both cases (a "weak" inner part and "strong" outer parts, or a "strong" inner part and "weak" outer parts).

\section{Asymptotic approach}

Finally the analysis is extended to an asymmetric rod with free ends where the densities and the Young's moduli of the components are dependent on the longitudinal coordinate. Since it is not possible to find the exact solution in this case, we implement a perturbation scheme.

Let us introduce a small parameter $\varepsilon=\frac{E_{12}}{h_{12}} \ll 1$ associated with contrast material properties of the components and consider the global low-frequency regime of the form $\lambda_{1} \sim \lambda_{2} \sim \lambda_{3} \sim \varepsilon$. The frequencies and the displacement are 
expanded as asymptotic series

$$
\begin{aligned}
\lambda_{i}^{2} & =\varepsilon\left(\lambda_{i 0}^{2}+\varepsilon \lambda_{i 1}^{2}+\varepsilon^{2} \lambda_{i 2}^{2}+\ldots\right), \\
u & =u_{0}+\varepsilon u_{1}+\varepsilon^{2} u_{2}+\ldots .
\end{aligned}
$$

For notational convenience we introduce the following dimensionless scaling

$$
\chi_{i}=\frac{x}{h_{i}}, \quad i=1,2,3,
$$

thus $\quad-1 \leq \chi_{1} \leq 1, \quad h_{12} \leq \chi_{2} \leq h_{12}+1, \quad-h_{13}-1 \leq \chi_{3} \leq-h_{13}$.

The equation of motion Eq.(1) can be rewritten as

$$
\frac{d}{d \chi_{i}}\left(\tilde{E}_{i} \frac{d u}{d \chi_{i}}\right)+\tilde{\rho}_{i} \lambda_{i}^{2} u=0, \quad i=1,2,3,
$$

with the following boundary value problem

$$
\begin{aligned}
u(-1+0) & =u\left(-h_{13}-0\right), \\
u(1-0) & =u\left(h_{12}+0\right), \\
\varepsilon E_{23} h_{32} u^{\prime}(-1+0) & =u^{\prime}\left(-h_{13}-0\right), \\
\varepsilon u^{\prime}(1-0) & =u^{\prime}\left(h_{12}+0\right), \\
u^{\prime}\left(-1-h_{13}\right) & =0, \\
u^{\prime}\left(1+h_{12}\right) & =0 .
\end{aligned}
$$

On substituting the expansion Eq.(50) into the equation of motion Eq.(51) at leading order $\varepsilon^{0}$ we infer

$$
\frac{d}{d \chi_{i}}\left(\tilde{E}_{i} \frac{d u_{0}}{d \chi_{i}}\right)=0, \quad i=1,3,
$$

subject to

$$
\begin{aligned}
u_{0}^{\prime}\left(-h_{13}-0\right) & =0, \\
u_{0}^{\prime}\left(h_{12}+0\right) & =0, \\
u_{0}^{\prime}\left(-1-h_{13}\right) & =0, \\
u_{0}^{\prime}\left(1+h_{12}\right) & =0 .
\end{aligned}
$$

Integrating the equation Eq. (53) we receive

$$
\frac{d u_{0}}{d \chi_{i}}=\frac{C_{i}}{\tilde{E}_{i}}, \quad i=1,3,
$$


135 where $C_{i}$ are constants.

From Eq.(55) and Eq.(54) it may be deduced that

$$
\begin{aligned}
& u_{0}=U_{L}, \quad-1-h_{13} \leq \chi_{3} \leq-h_{13}, \\
& u_{0}=U_{R}, \quad h_{12} \leq \chi_{2} \leq 1+h_{12},
\end{aligned}
$$

where $U_{R}$ and $U_{L}$ are constants of integration.

The leading order problem for the displacement of the inner part is given by

$$
\frac{d}{d \chi_{1}}\left(\tilde{E}_{1} \frac{d u_{0}}{d \chi_{1}}\right)=0,
$$

with

$$
\begin{aligned}
u_{0}(-1) & =U_{L}, \\
u_{0}(1) & =U_{R} .
\end{aligned}
$$

Integrating Eq.(57) over $-1 \leq \chi_{1} \leq 1$ and employing Eq.(58) we result in

$$
u_{0}=\int_{-1}^{\chi_{1}} \frac{U_{R}-U_{L}}{\tilde{E}_{1} e_{1}} d \chi_{1}, \quad-1 \ll \chi_{1} \ll 1,
$$

where

$$
e_{1}=\int_{-1}^{1} \tilde{E}_{1}^{-1} d \chi_{1}
$$

It is worth noting that the leading order displacement (56), (59) correlates with Eq.(15) obtained for a symmetric rod, see Fig. 3.

At next order $\varepsilon^{1}$ the analysis for the outer parts gives estimates for frequencies and a relation between $U_{L}$ and $U_{R}$. The problem is formulated as follows

$$
\frac{d}{d \chi_{i}}\left(\tilde{E}_{i} \frac{d u_{1}}{d \chi_{i}}\right)+\tilde{\rho}_{i} \lambda_{i 0}^{2} u_{0}=0, \quad i=1,3
$$

subject to

$$
\begin{aligned}
u_{1}^{\prime}\left(-h_{13}-0\right) & =E_{23} h_{32} U_{L}, \\
u_{1}^{\prime}\left(h_{12}+0\right) & =U_{R}, \\
u_{1}^{\prime}\left(-1-h_{13}\right) & =0, \\
u_{1}^{\prime}\left(1+h_{12}\right) & =0 .
\end{aligned}
$$


Integrating the equation Eq.(60) over $h_{12} \leq \chi_{2} \leq 1+h_{12}$ and using Eq.(56) for $u_{0}$ one arrives at

$$
\left.\tilde{E}_{2} \frac{d u_{1}}{d \chi_{2}}\right|_{\chi_{2}=1+h_{12}}-\left.\tilde{E}_{2} \frac{d u_{1}}{d \chi_{2}}\right|_{\chi_{2}=h_{12}}=-\lambda_{20}^{2} U_{R} r_{2},
$$

where

$$
r_{2}=\int_{h_{12}}^{1+h_{12}} \tilde{\rho}_{2} d \chi_{2} .
$$

Employing the conditions Eq.(61), we deduce the expression for $\lambda_{20}^{2}$ in the form

$$
\lambda_{20}^{2}=\frac{\tilde{E}_{2}(1)\left(U_{R}-U_{L}\right)}{\tilde{E}_{1}(1) U_{R} e_{1} r_{2}} .
$$

Similarly the expression for $\lambda_{30}^{2}$ is found as

$$
\lambda_{30}^{2}=\frac{\tilde{E}_{3}(-1)\left(U_{L}-U_{R}\right) E_{23} h_{32}}{\tilde{E}_{1}(-1) U_{L} e_{1} r_{3}},
$$

where

$$
r_{3}=\int_{-1-h_{13}}^{-h_{13}} \tilde{\rho}_{3} d \chi_{3}
$$

Using the relation $\lambda_{2}=h_{23} c_{32} \lambda_{3}$ and Eq.(64), it is possible to express $\lambda_{20}^{2}$ as

$$
\lambda_{20}^{2}=\frac{\tilde{E}_{3}(-1)\left(U_{L}-U_{R}\right) h_{23} \rho_{23}}{\tilde{E}_{1}(-1) U_{L} e_{1} r_{3}} .
$$

Therefore from Eq. (63) and Eq.(65) we obtain the solution $\lambda_{20}^{2}=0$ associated with the rigid body motion and

$$
\lambda_{20}^{2}=\frac{1}{e_{1}}\left(\frac{\tilde{E}_{2}(1)}{\tilde{E}_{1}(1) r_{2}}+\frac{\tilde{E}_{3}(-1) \rho_{23} h_{23}}{\tilde{E}_{1}(-1) r_{3}}\right),
$$

for which

$$
U_{L}=-U_{R} \rho_{23} h_{23} \frac{\tilde{E}_{1}(1)}{\tilde{E}_{2}(1)} \frac{\tilde{E}_{3}(-1)}{\tilde{E}_{1}(-1)} \frac{r_{2}}{r_{3}} .
$$

In case when $E_{i}$ and $\rho_{i}$ are independent of $\chi_{i}$ Eq. (66), Eq. (67) reduce to

$$
\lambda_{20}^{2}=\frac{1}{2}\left(1+\rho_{23} h_{23}\right),
$$

for which

$$
U_{L}=-U_{R} \rho_{23} h_{23},
$$


respectively.

In case of the symmetric rod considered previously in Section 3.1, $\rho_{23}=1$, $h_{23}=1$ and

$$
\lambda_{20}^{2}=1
$$

with

$$
U_{L}=-U_{R}
$$

which matches the previous results, see Eq. (15).

\section{Conclusion}

The low-frequency vibrations of a three-component elastic rod with contrast properties have been studied. It is demonstrated that a contrast may result in the lowest natural frequencies being close to zero. A classification involving global and local low-frequency regimes is presented. The conditions on the problem parameters (14), corresponding to the global low-frequency behavior are derived.

It could be suggested from the consideration above that the quasi-static linear displacement variations occur when a "stronger" part of the system performs almost rigid body motion, with the "weaker" parts undergoing almost homogeneous deformations. At the same time the constraints due to a "weaker" part should not allow proper rigid body motion corresponding to the zero natural frequency.

Generally speaking, the developed methodology can be regarded as a lowfrequency perturbation of rigid body motions, similarly to ideas expressed in 16] for a viscoelastic bar with no assumption on the contrast. In the case of variable problem parameters, see Section 5, the perturbation procedure does not rely on explicit analytical solution.

The obtained results have a clear relation to evaluation of the lowest thickness resonance frequencies [10] of layered plates and shells. The approach can be expanded to more sophisticated formulations for structures with contrast material and geometric properties. In a more general setup the solution procedure 
should apparently include numerical routines at least for "weaker" parts of the studied structures.

\section{References}

[1] C. Horgan, A. Chan, Vibration of inhomogeneous strings, rods and membranes, Journal of Sound and Vibration 225 (3) (1999) 503-513.

[2] I. Elishakoff, Eigenvalues of inhomogeneous structures: unusual closed-form solutions, CRC Press, 2004.

[3] D. Zenkert, An introduction to sandwich structures, EMAS Ltd., London, 1995.

[4] S. Sorokin, Analysis of wave propagation in sandwich plates with and without heavy fluid loading, Journal of Sound and Vibration 271 (2004) 10391062 .

[5] C. Chapman, An asymptotic decoupling method for waves in layered media, in: Proceedings of the Royal Society of London Series A, Vol. 469, The Royal Society, 2013, p. 20120659.

[6] T. P. Martin, C. N. Layman, K. M. Moore, G. J. Orris, Elastic shells with high-contrast material properties as acoustic metamaterial components, Physical Review B 85 (2012) 161103.

[7] J. Helsing, R. S. McPhedran, G. W. Milton, Spectral super-resolution in metamaterial composites, New Journal of Physics 13 (11) (2011) 115005.

[8] K. Cherednichenko, V. Smyshlyaev, V. Zhikov, Nonlocal homogenized limits for composite media with highly anisotropic periodic fibres, Proceedings of the Royal Society of Edinburgh: Section A Mathematics 136 (01) (2006) $87-114$.

[9] V. Smyshlyaev, Propagation and localization of elastic waves in highly anisotropic periodic composites via two-scale homogenization, Mechanics of Materials 41 (2009) 434-447. 
[10] J. Kaplunov, L. Kossovich, E. Nolde, Dynamics of thin walled elastic bodies, Academic Press, 1998.

[11] M. Lutianov, G. Rogerson, Long wave motion in layered elastic media, International Journal of Engineering Science 48 (12) (2010) 1856-1871.

[12] A. K. Noor, W. S. Burton, Assessment of shear deformation theories for multilayered composite plates, Applied Mechanics Reviews 42 (1) (1989) $1-13$.

[13] A. Goldenveizer, J. Kaplunov, E. Nolde, On Timoshenko-Reissner type theories of plates and shells, International Journal of Solids and Structures 30 (5) (1993) 675-694.

[14] K. Le, Vibrations of shells and rods, Springer Berlin, 1999.

[15] M. Cherdantsev, K. Cherednichenko, Homogenisation in finite elasticity for composites with a high contrast in the vicinity of rigid-body motions, arXiv preprint arXiv:1303.1224v.

[16] J. Kaplunov, A. Shestakova, I. Aleynikov, B. Hopkins, A. Talonov, Lowfrequency perturbations of rigid body motions of a viscoelastic inhomogeneous bar, Mechanics of Time-Dependent Materials (2015) 1-17. 